\title{
Complete Resolution of a Case of Calcific Tendinitis of the Longus Colli with Conservative Treatment
}

\author{
Fumitake Tezuka ${ }^{1}$, Toshinori Sakai ${ }^{1}$, Ryo Miyagi ${ }^{1}$, Yoichiro Takata ${ }^{1}$, \\ Kosaku Higashino ${ }^{1}$, Shinsuke Katoh ${ }^{1}$, Koichi Sairyo ${ }^{2}$, Natsuo Yasui ${ }^{1}$ \\ ${ }^{1}$ Department of Orthopedics, Institute of Health Biosciences, The University of Tokushima Graduate School, Tokushima, Japan \\ ${ }^{2}$ Department of Orthopedics, Teikyo University Mizonokuchi Hospital, Tokyo, Japan
}

Acute calcific tendinitis of the longuscolli is a self-limiting inflammatory condition caused by calcium hydroxyapatite deposition in the longuscolli tendon. Although several case reports have described its radiological presentation, few reports provide detailed chronological accounts through symptomatic and radiologic resolution. A 59-year-old woman presented with severe neck pain and stiffness of a few days duration as well as moderate discomfort when swallowing. Lateral radiographs revealed a large calcium deposit anterior to the $\mathrm{C1}-\mathrm{C} 2$ joint and swelling of the prevertebral soft tissue from C1 to C5. CT and magnetic resonance imaging showed fluid in the retropharyngeal gap.A soft collar and non-steroidal anti-inflammatory drug were prescribed, without antibiotics. At 4 months after presentation, the calcium deposit and all symptoms had resolved completely. Although this disease is comparatively rare, physicians should keep it in mind when a patient presents with acute severe neck pain.

Keywords: Calcific tendinitis of the longuscolli; Conservative treatment

\section{Introduction}

Acute calcific tendinitis of the longuscolli, which is also known as acute calcific prevertebral tendinitis or retropharyngeal tendinitis, is a self-limiting inflammatory condition caused by calcium hydroxyapatite deposition in the longuscolli tendon. Hartley [1] reported the first case in 1964, which involved acute upper cervical pain associated with a large amorphous calcium deposit just anterior to the atlanto-axial joint. Ring et al. [2] subsequently reported that initial misdiagnosis of the diseasehad led to unnecessary medical treatment in five patients and unnecessary open biopsy in one patient. Recently,
Offiah and Hall [3] asserted that avoiding unnecessary medical and surgical interventions relies on identifying the presence of the disease as well as being aware of its radiological appearance, particularly on computed tomography (CT). Although the literature contains several case reports describing its radiological presentation, few reports provide detailed chronological accounts through symptomatic and radiologic resolution.

In this report, we present a case of acute calcific tendinitis of the longuscolli, describing in detail the symptomsas well as detailed radiological findings chronologically, including plain X-ray, $\mathrm{CT}$, and magnetic resonance imaging (MRI).

Received Apr 14, 2013; Revised Jul 7, 2013; Accepted Jul 8, 2013

Corresponding author: Fumitake Tezuka

Department of Orthopedics, Institute of Health Biosciences, The University of Tokushima Graduate School,

3-18-15 Kuramoto-cho, Tokushima 770-8503, Japan

Tel: +81-88-633-7240, Fax: +81-88-633-0178, E-mail:m01059ft@gmail.com 


\section{Case report}

A 59-year-old woman presented with severe neck pain and stiffness of a few days duration as well as moderate discomfort when swallowing. She had no trauma and no current or recent febrile upper respiratory tract infections. On physical examination, swelling and tenderness of the sternocleidomastoid was found bilaterally. Neck pain was induced by extension and reduced by flexion. Range of motion (ROM) of the cervical spine was extremely limited. Visual inspection of the pharynx and ultrasound examination of her neck revealed no pharyngitis, goiter, or lymphadenitis. Her blood pressure, pulse, respiratory rate, and temperature were within normal limits. White blood cell count was 6,160 cells/ $\mathrm{mm}^{3}$ and C-reactive protein level was $1.2 \mathrm{mg} / \mathrm{dL}$. Lateral radiographs of the cervical spine revealed a large calcium deposit anterior to the $\mathrm{C} 1-\mathrm{C} 2$ joint and swelling of the prevertebral soft tissue from C1 to C5 (Fig. 1A). Neck CT scans revealed a low density area with the appearance of a convex lens in the retropharyngeal gap, but the contours of the longuscolli and longuscapitis muscles were clear (Fig. 1B). MRI showed fluid in the retropharyngeal gap between $\mathrm{C} 1$ and C6 (Fig. 2). There were no findings of spondylitis, epidural abscess, or tumor.

A diagnosis of acute calcific tendinitis of the longuscolli was made, and a soft collar and non-steroidal antiinflammatory drug (NSAID) were prescribed, without antibiotics. Two weeks later, neck pain, dysphagia, and ROM limitation were all reduced, and plain X-ray films taken at 3 weeks after presentation revealed reduced retropharyngeal soft-tissue swelling and almost complete disappearance of the amorphous calcification anterior to C1-C2 (Fig. 3). At 4 months after presentation, the cal-
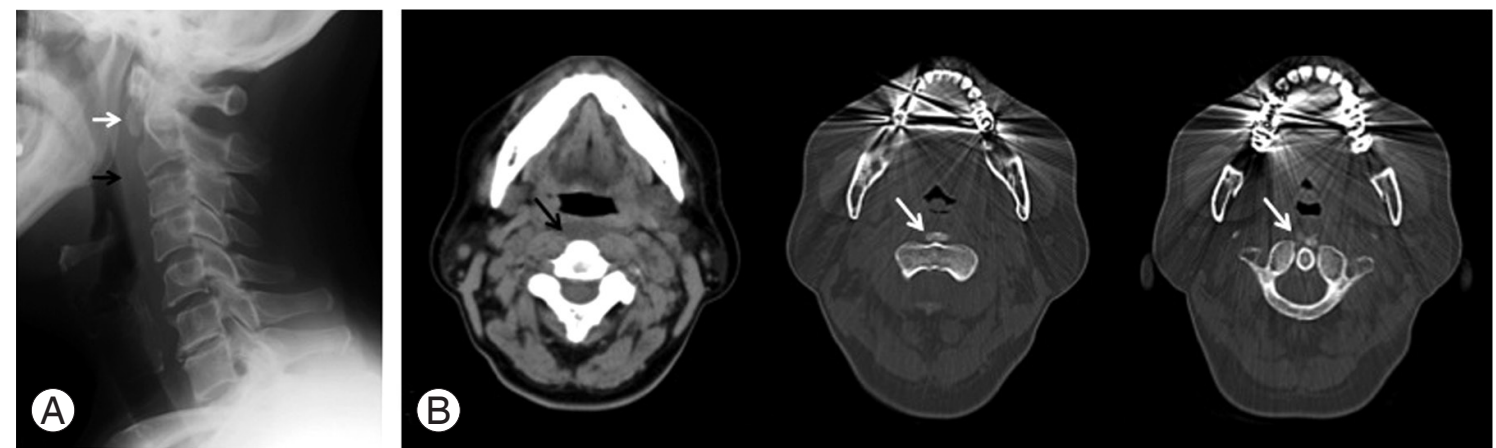

Fig. 1. Radiological findings on initial presentation. (A) Lateral radiograph of the cervical spine reveals a large calcium deposit anterior to the C1-C2 joint (white arrow) and prevertebral soft-tissue swelling from C1 to C5 (black arrow). (B) Neck computed tomography shows a low density area, like a convex lens, in the retropharyngeal gap (black arrow) andacalcium deposit anterior to the C1-C2 joint (white arrows).
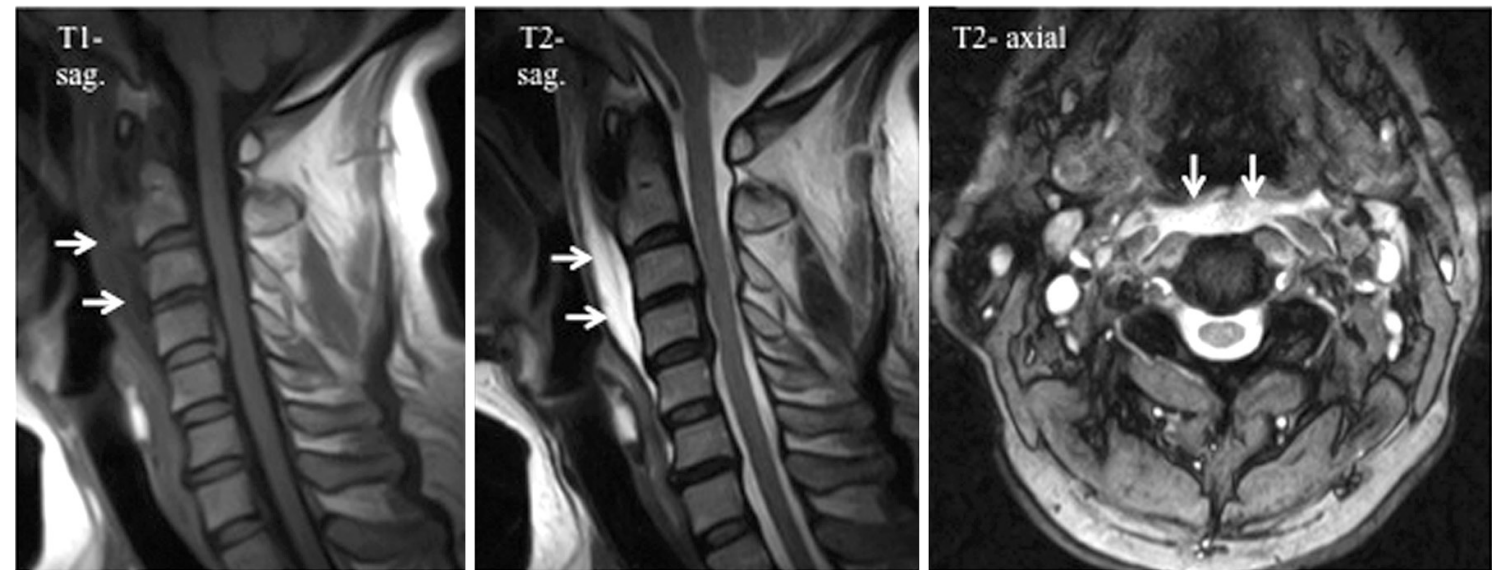

Fig. 2. Magnetic resonance imaging shows fluid in the retropharyngeal space between C1 and C6 (white arrows). sag., sagittal. 


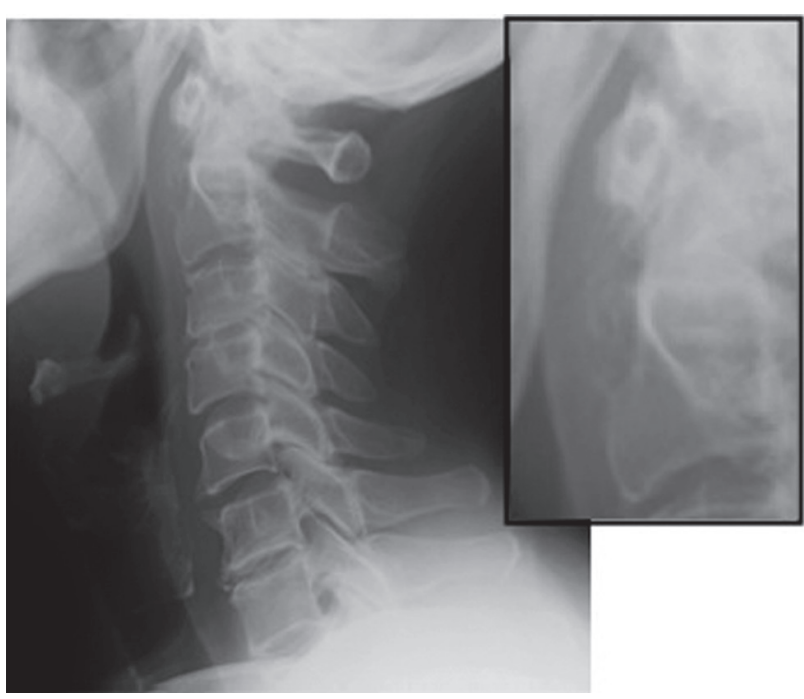

Fig. 3. Three weeks after presentation, much of the retropharyngeal soft-tissue swelling had reduced in size, but the calcium deposit was still detectable. cium deposit and all of the abovementioned symptoms had resolved completely (Fig. 4). At 6months, fluid in the retropharyngeal gap between $\mathrm{C} 1$ and $\mathrm{C} 6$ was no longer evident on MRI (Fig. 5).

\section{Discussion}

Acute calcific tendinitis of the longuscolli is among the diseases in which calcium hydroxyapatite crystals are deposited in large joints, in this case the shoulder joint. It is an inflammatory condition caused by the deposition in the superior oblique tendon fibers of the longuscolli muscles, mainly at the $\mathrm{C} 1-\mathrm{C} 2$ level $[2,4]$. The etiology of the deposition is still unclear, but repetitive trauma, recent injury, tissue necrosis, or ischemia may play a role [5].

Symptoms peculiar to this disease, which are secondary to inflammation of the longuscolli muscle, are 1) acute to subacute onset of neck pain, 2) dysphagia, odynophagia,
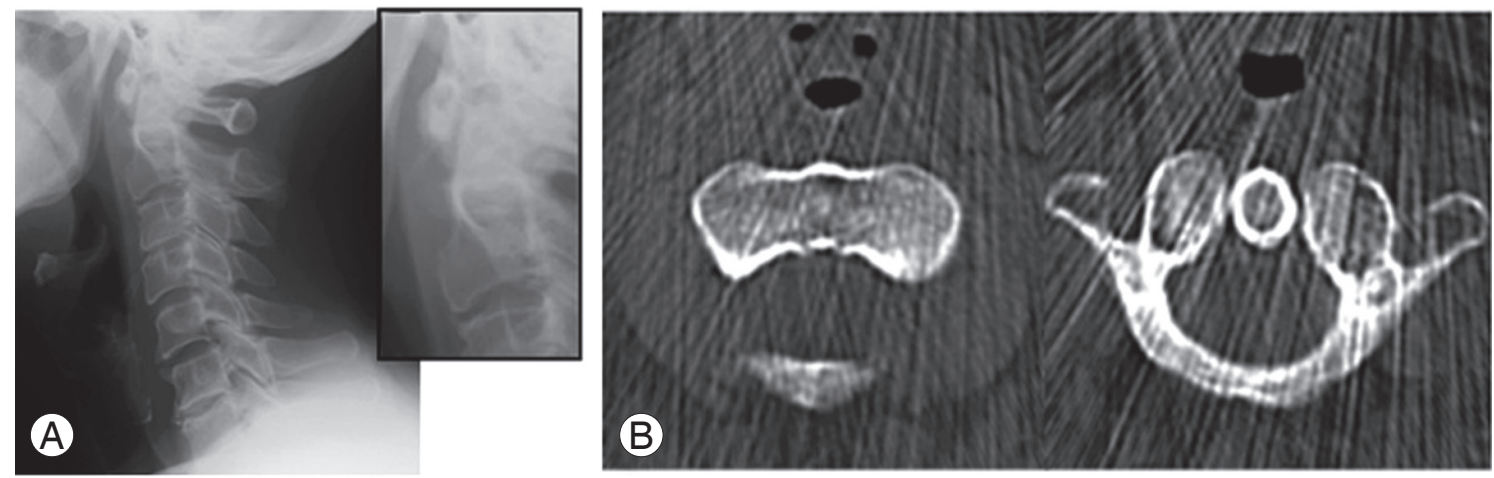

Fig. 4. Four monthsafter presentation, (A) a lateral radiograph shows no evidence of the calcium deposit and (B) computed tomography confirms the calcium deposit had resolved completely.
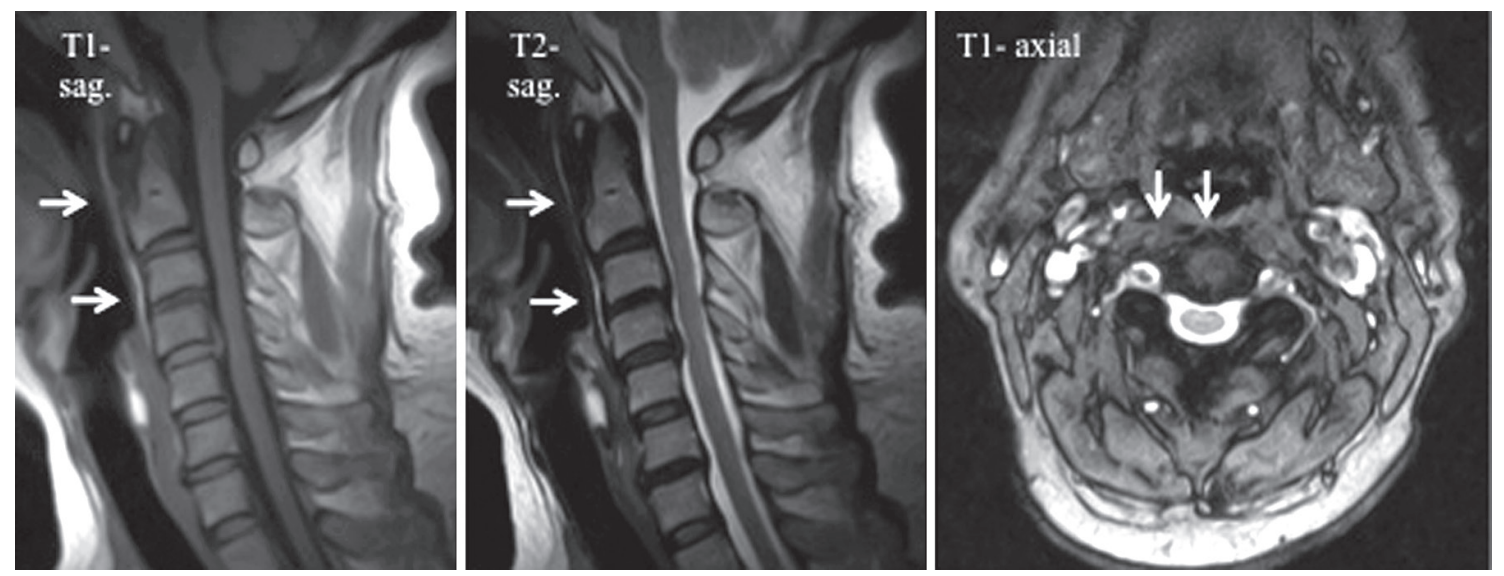

Fig. 5. Magnetic resonance imaging at 6 months after presentation shows no fluid in the retropharyngeal gap between C1 and C6 (white arrows). sag., sagittal. 
and 3) low-grade fever. Adults between 30 and 60 years of age are mostly susceptible to this disease [2,4]. Dysphagia is caused by the proximity between the retropharyngeal space and the pharyngeal constrictors [3]. Typically cervical paraspinal muscle spasms are found on slight flexion. Neck ROM is extremely limited due to severe pain, especially on extension. Sometimes there is tenderness to palpation anterolaterally or posterolaterally, but not directly posteriorly, over the spinous process. The tenderness caused by deep anterior palpation between the larynx and sternocleidomastoid muscle might be due to direct manipulation of the inflamed longuscolli muscle [2]. Laboratory tests show a mildly elevated white blood cell count, C-reactive protein level, or erythrocyte sedimentation rate $[2,4]$. Since these clinical and biological findings are not specific to this condition, retropharyngeal abscess or cervical spondylodiscitis should be ruled out.

Typical radiological findings are prevertebral soft-tissue swelling and amorphous calcification anterior to $\mathrm{C} 1-\mathrm{C} 2$. Diffuse prevertebral soft tissue thickening extends from $\mathrm{C} 1$ to $\mathrm{C} 4$, even though it can extend as inferiorly as C6 [6]. In a small percentage of patients, calcification either is not detectable or is insufficient for accurate diagnosis on lateral radiographs $[2,4]$. In such cases, CT is necessary to confirm the location of any areas of calcification in the longuscolli tendons and to demonstrate soft-tissue swelling in the retropharyngeal space $[3,4,7,8]$. Fortunately, in the present case, characteristic findings were evident on lateral radiographs, and CT scans provided useful information for identifying calcific deposits. Generally, MRI is not necessary to diagnose the condition, especially as it is not sufficiently sensitive to detect calcification of the tendon, but it is useful for detecting inflammation around the longuscolli muscle and to help exclude spondylitis, epidural abscess, and tumorous lesion.

This disease is thought to be a self-limiting condition that resolves spontaneously after 1-2 weeks. Invasive examinations and surgical intervention should be avoided as much as possible, particularly as anti-inflammatory medications, analgesics, and immobilization typically provide symptomatic relief. In the present case, we were able to diagnose the disease from the characteristic radiological findings, and with conservative treatment using NSAIDs and a soft collar, the patient's symptoms and radiological findings were resolved.

A review of the English-language literature for cases of acute calcific tendinitis of the longuscolli $[1,5,6,9,10]$
Table 1. When was the calcium deposit absorbed?

Duration

No. of patients

Less than 1 month 11

After 1 month less than 2 months 4

After 2 months

2

Using 17 patients' radiographic (X-ray or computed tomography) from 14 literatures.

yielded data on 17 patients about the time to absorption of calcium deposits, as evidenced on plain X-ray films or CT scans (Table 1). In the present case, more than 3 weeks was required for complete resolution. Although symptoms are not always synchronous with their radiological findings, on the basis of this literature review, 1-2 months may be needed for complete healing.

We have reported a case of acute calcific tendinitis of the longuscolli that was completely resolved by conservative treatment. Although the disease is a comparatively rare, physicians should keep it in mind when a patient presents with acute severe neck pain. The differential diagnosis should rule out acute purulent diseases such as retropharyngeal abscess and pyrogenic spondylitis, trauma, and neoplastic disease.

Agreement was obtained from the patient to report her data for publication.

\section{Conflict of Interest}

No potential conflict of interest relevant to this article was reported.

\section{References}

1. Hartley J. Acute cervical pain associated with retropharyngeal calcium deposit. a case report. J Bone Joint Surg Am 1964;46:1753-4.

2. Ring D, Vaccaro AR, Scuderi G, Pathria MN, Garfin SR. Acute calcific retropharyngeal tendinitis. Clinical presentation and pathological characterization. J Bone Joint Surg Am 1994;76:1636-42.

3. Offiah CE, Hall E. Acute calcific tendinitis of the longus colli muscle: spectrum of CT appearances and anatomical correlation. Br J Radiol 2009;82:e117-21.

4. Eastwood JD, Hudgins PA, Malone D. Retropharyngeal effusion in acute calcific prevertebral tendinitis: 
diagnosis with CT and MR imaging. AJNR Am J Neuroradiol 1998;19:1789-92.

5. De Maeseneer M, Vreugde S, Laureys S, Sartoris DJ, De Ridder F, Osteaux M. Calcific tendinitis of the longus colli muscle. Head Neck 1997;19:545-8.

6. Fahlgren H. Retropharyngeal tendinitis. Cephalalgia 1986;6:169-74.

7. Artenian DJ, Lipman JK, Scidmore GK, Brant-Zawadzki M. Acute neck pain due to tendonitis of the longus colli: CT and MRI findings. Neuroradiology
1989;31:166-9.

8. Omezzine SJ, Hafsa C, Lahmar I, Driss N, Hamza H. Calcific tendinitis of the longus colli: diagnosis by CT. Joint Bone Spine 2008;75:90-1.

9. Haun CL. Retropharyngeal tendinitis. AJR Am J Roentgenol 1978;130:1137-40.

10. Newmark H 3rd, Forrester DM, Brown JC, Robinson A, Oiken SM, Bledsoe R. Calcific tendinitis of the neck. Radiology 1978;128:355-8. 